\title{
中国抖学
}

\section{仅用一个采样点和自相关函数恢复离散信号 的唯一性定理及其算法}

吴忠泽李衍达常㛠

（清华大学自动化系,北京）

\section{摘要}

本文是文献 [1] 续篇. 对于仅用一个采样点以及自相关函数恢复整个离散信号 的唯一性问题，本文提出了四个定理，并作了详细的证明; 还给出了实现这种恢复的 一种时域迭代算法,大量实例表明这种算法是十分有效的.

\section{关键词：离散信号理论,唯一性理论,时域迭代法实现}

\section{一、引 言}

信号处理的一个基本问题是在噪声背景中恢复信号. 因而, 近年来关于信号重构问题的 理论研究一直十分活跃. 在大量的实际问题中, 也往往是仅仅已知部分信息, 却要求恢复整个 离散信号. 例如, 在雷达信号处理中,通常接受的只是 8-16 点反射序列, 而不知全部; 在地震 信号处理中, 虽然子波的相谱是难以估计的, 然而由于地层反射系数近似于白噪, 因此能比较 容易获得子波的幅度谱 ${ }^{[2]}$.

从部分信息恢复信号需要解决两个主要问题. 第一,要知道多少信息才能完全恢复信号? 在什么条件下,可以用较少的信息唯一恢复信号?第二,提出恢复信号的实用算法. 在文献 [1] 中, 曾经在 Nawab 等人工作的基础上 ${ }^{[3]}$, 提出了利用幅谱和时域中的部分信息来恢复非最 小相位离散信号的三个唯一性定理及其算法一三三域迭代法。在这些定理中，规定了已知段 的起始点可以是未知序列中的任何一个采样点(而不必限制在起始点 $x(0)$ ), 它的长度 $m$ 也可 以小于未知序列长度 $N$ 的一半 (即 $m=N / 3-2 N / 3$ ), 从而扩展了 $\mathrm{Nawab}$ 等人提出的定理 的适用范围。

本文在此基础上,进一步利用了全部的自相关函数 (即全部幅度谱信息) 作为恢复未知序 列的约束条件，一方面从理论上证明了只要满足一定的条件，那末恢复非最小相位信号所需已 知段的长度 $m$ 就可以缩小到 $m=1$, 即仅用一个已知采样点(也不必限制在起始点) 以及自相 关函数就可以恢复任意一个有限长度离散信号, 为此提出了四个唯一性定理; 另一方面, 还给 出了实现这种恢复的一种时域迭代法. 这种算法类似于可变多面体的搜索方法, 本文用大量 
实例表明,这种算法是十分有效的.

\section{二、唯一性定理}

考虑一个实的混合相位离散时间信号 $x(n)$, 在时间间隔 $0 \leqslant n \leqslant N-1$ 之外, 其值全为 零. 它的自相关函数为 $R_{j}(0 \leqslant j \leqslant N-1)$. 下面, 我们就来详细讨论一下仅用一个采样点 以及自相关函数来恢复整个未知信号 $x(n)$ 的唯一性问题.

\section{1. 首先讨论已知采样点是原始序列的起始点 $x(0)$ 时的情形}

定义 1. 令向量值函数 $F$ 为定义在区域 $\mathscr{D} \subset R^{N-1}$ 上而取值于 $R^{N}$ 的非线性实值函数。简 记为 $F: \mathscr{D} \subset R^{N-1} \rightarrow R^{1}$, 它的具体形式为:

$$
F(X)=\left[f_{1}(X), f_{2}(X), \cdots, f_{N}(X)\right]^{T},
$$

其中, $X$ 为末知采样值 $x_{1}, x_{2}, \cdots, x_{N-1}$ 构成的向量: $X=\left[x_{1}, x_{2}, \cdots, x_{N-1}\right]^{T} \in R^{N-1} ; f_{i}(X)$ $(i=1,2, \cdots, N)$ 为定义在区域 $\mathscr{D} \subset R^{N-1}$ 上而取值于 $R^{1}$ 的非线性实值函数. 简记为 $f_{i}:$ $\mathscr{D} \subset R^{N-1} \rightarrow R^{1}$, 它们的具体形式为:

$$
\left\{\begin{array}{l}
f_{1}(X)=x_{0}^{2}+x_{1}^{3}+\cdots+x_{N-1}^{2}-R_{0}, \\
f_{2}(X)=x_{0} x_{1}+x_{1} x_{2}+\cdots+x_{N-2} x_{N-1}-R_{1}, \\
f_{3}(X)=x_{0} x_{2}+x_{1} x_{2}+\cdots+x_{N-3} x_{N-1}-R_{2}, \\
\cdots \cdots \cdots \cdots \cdots \\
f_{N-1}(X)=x_{0} x_{N-2}+x_{1} x_{N-1}-R_{N-2}, \\
f_{N}(X)=x_{0} x_{N-1} R_{N-1} .
\end{array}\right.
$$

这里, $R_{i}(0 \leqslant j \leqslant N-1)$ 为序列 $x(n)$ 的自相关函数通常可由已知的幅度谱 $|X(\omega)|$ 的逆 Fourier 变换求得; $x_{0}$ 为已知端点值; $x_{1}, x_{2}, \cdots, x_{N-1}$ 为序列 $x(n)$ 的未知采样值.

定义 2. 令目标函数 $\varphi(X)=F(X)^{T} \cdot F(X)$ 为定义在区域 $\mathscr{D} \subset R^{N-1}$ 上而取值于 $R^{1}$ 的 非线性实值函数。简记为 $\varphi: \mathscr{D} \subset R^{N-1} \rightarrow R^{1}$.

定理 1. 对于 $N>0$, 令 $x(n)$ 是一个在 $0 \leqslant n \leqslant N-1$ 时间间隔之外为 0 的实的混合 相位序列. 假设 $x(0) \neq 0$, 那末端点 $x(0)$ 以及自相关 $R_{i}(0 \leqslant j \leqslant N-1)$ 唯一地确定了 整个序列 $x(n)$ ，当目标函数 $\varphi(X)$ 是凸集合 $\mathscr{D}_{0} \subset \mathscr{D}$ 上的严格凸函数.

证. 由定义 2 可知, 目标函数

$$
\varphi(X)=F(X)^{T} \cdot F(X) .
$$

将定义 $1, F(X)=\left(f_{1}(X), f_{2}(X), \cdots, f_{N}(X)\right)^{T}$ 代人 $(1)$ 式可得

$$
\varphi(X)=f_{1}(X)+f_{2}^{2}(X)+\cdots+f_{N}^{2}(X) \geqslant 0 .
$$

鉴于 $x(0)$ 和 $R_{i}$ 的相容关系

$$
R(j)=\sum_{k=0}^{N-1-i} x(k) \cdot x(j+k),
$$

必存在 $X^{*}$, 使 $\Phi \varphi\left(X^{*}\right)=0$.

又因为目标函数 $\varphi(X)$ 是凸集合 $\mathscr{D}_{0} \subset \mathscr{D}$ 上的严格凸函数,所以函数 $\varphi(X)$ 在 $\mathscr{D}_{0}$ 上必存 唯一的全局极小点 $\widetilde{X}^{[3]}$, 也即

$$
\widetilde{X}=X^{*}
$$


故

因此,必有

$$
\varphi(\widetilde{X})=f_{1}(\widetilde{X})+f_{2}^{2}(\widetilde{X})+\cdots+f_{N}^{2}(\widetilde{X}) \equiv 0
$$

$$
\left\{\begin{array}{c}
f_{1}(\tilde{X}) \equiv 0 \\
f_{2}^{2}(\widetilde{X}) \equiv 0 \\
\vdots \\
f_{N}^{2}(\tilde{X}) \equiv 0
\end{array}\right.
$$

将(3)式代人定义 1 可得

$$
\left\{\begin{array}{l}
\left(x_{0}^{2}+\tilde{x}_{1}^{2}+\tilde{x}_{2}^{2}+\cdots+\tilde{x}_{N-1}^{2}-R_{0}\right)^{2} \equiv 0, \\
\left(x_{0} \tilde{x}_{1}+\tilde{x}_{1} \tilde{x}_{2}+\cdots+\tilde{x}_{N-2} \tilde{x}_{N-1}-R_{1}\right)^{2} \equiv 0, \\
\left(x_{0} \tilde{x}_{2}+\tilde{x}_{1} \tilde{x}_{3}+\cdots+\tilde{x}_{N-3} \tilde{x}_{N-1}-R_{2}\right)^{2} \equiv 0, \\
\left.\cdots \cdots \cdots+\tilde{x}_{1} \tilde{x}_{N-1}-R_{N-2}\right)^{2} \equiv 0, \\
\left(x_{0} \tilde{x}_{N-2}+\tilde{x}_{N-1}\right)^{2} \equiv 0 .
\end{array}\right.
$$

这意昧着,如下非线性方程组:

$$
\begin{aligned}
& x_{1}^{2}+x_{2}^{2}+\cdots+x_{N-1}^{2}=R_{0}-x_{0}^{2}, \\
& \left\{\begin{array}{l}
x_{0} x_{1}+x_{1} x_{2}+\cdots+x_{N-2} x_{N-1}=R_{1}, \\
x_{0} x_{2}+x_{1} x_{3}+\cdots+x_{N-3} x_{N-1}=R_{2}, \\
\cdots \cdots \cdots+x_{1} \\
x_{0} x_{N-1}+x_{N-1}=R_{N-2}, \\
x_{0} x_{N-1}=R_{N-1}
\end{array}\right.
\end{aligned}
$$

必存在唯一解

$$
X=\left[\tilde{x}_{1}, \tilde{x}_{2}, \ldots, \tilde{x}_{N-1}\right]^{T} .
$$

考虑到上述方程组中的 $R_{j}(0 \leqslant j \leqslant N-1)$ 为序列 $x(n)$ 的自相关函数 (通常它可由已知 幅度谱 $|X(\omega)|$ 的逆 Fourier 变换求得)， $x_{0}$ 为已知端点值. 所以当目标函数 $\varphi(X)$ 是凸集 合 $\mathscr{D}_{0} \subset \mathscr{D}$ 上的严格凸函数时, 仅用 $x(0)$ 和 $R_{i}(0 \leqslant j \leqslant N-1)$ 就能唯一地确定未知采 样值 $x_{1}, x_{2}, \cdots, x_{N-1}$, 即整个序列 $x(n)$.

证毕.

定理 2. 对于 $N>0$, 令 $x(n)$ 是一个在 $0 \leqslant n \leqslant N-1$ 时间间隔之外为 0 的实的非偶序 列, 假设 $x(0) \neq 0$, 并满足 $x(N-1)=x(0)$, 那末仅用端点 $x(0)$ 以及幅度谱 $|X(\omega)|$ 不能 唯一地恢复原始序列 $x(n)$, 它们至少还确定了另一个不同的序列 $y(n)$, 并且满足关系式

$$
y(n)=x(N-1-n) \text {. }
$$

证. 设 $x(n)$ 的 $Z$ 变换 $X(Z)$ 的零点为 $Z_{1}, Z_{2}, \cdots, Z_{N-1}$. 由代数方程的根与系数的关 系 ${ }^{[4]}$ 可知

$$
Z_{1} Z_{2} \cdots Z_{N-1}=(-1)^{N-1} \frac{x(0)}{x(N-1)},
$$

根据已知条件 $x(N-1)=x(0) \neq 0$ 可得

$$
Z_{1} Z_{2} \cdots Z_{N-1}=(-1)^{N-1} \text {, }
$$

两边取共轭 


$$
Z_{1}^{*} Z_{2}^{*} \cdots Z_{N-1}^{*}=(-1)^{N-1}
$$

再取倒数

$$
\frac{1}{Z_{1}^{*} Z_{2}^{*} \cdots Z_{N-1}^{*}}=(-1)^{-(N-1)}
$$

由(5)式和(6)式可得

$$
Z_{1} Z_{2} \cdots Z_{N-1}=\frac{1}{Z_{1}^{*} Z_{2}^{*} \cdots Z_{N-1}^{*}}
$$

我们不妨就取零点 $\frac{1}{Z_{1}^{*}}, \frac{1}{Z_{2}^{*}}, \cdots, \frac{1}{Z_{N-1}^{*}}$ 作为 $Y(Z)$ 的全部零点,即

$$
Y(Z)=x(0) \cdot\left(Z-\frac{1}{Z_{1}^{*}}\right)\left(Z-\frac{1}{Z_{2}^{*}}\right) \cdots\left(Z-\frac{1}{Z_{N-1}^{*}}\right),
$$

而已设

$$
X(Z)=x(N-1) \cdot\left(Z-Z_{1}\right)\left(Z-Z_{2}\right) \cdots\left(Z-Z_{N-1}\right),
$$

显而易见, $Y(Z)$ 与 $X(Z)$ 的全部零点一一构成共轭倒数对, 故由代数方程的根与系数的关系 可知

$$
y(n)=x(N-1-n),
$$

显然, $y(n)$ 与 $x(n)$ 不但具有相同的幅度谱,即

$$
|Y(\omega)|=|X(\omega)|,
$$

而且由(8)式可知,两个端点值也相同, 即

$$
y(0)=x(0) .
$$

考虑到 $x(n)$ 是一个实的非偶序列,即]

$$
x(n) \neq x(N-1-n),
$$

故

$$
y(n) \neq x(n) .
$$

所以, 当 $x(N-1)=x(0)$ 时, 仅用 $x(0)$ 和 $|X(\omega)|$ 是不能唯一恢复原始序列 $x(n)$ 的, 它们至少同时还确定了另一个不同于 $x(n)$ 的序列

$$
y(n)=x(N-1-n) .
$$

除了这个新序列 $y(n)$ 以外, 在某些条件下, 当 $x(N-1)=x(0)$ 时, 仅用 $x(0)$ 和 $|X(\omega)|$ 还能 确定另外若干个不同的序列. 定理 2 得证.

定理 3(充分条件). 对于 $N>0$, 令 $x(n)$ 是一个在 $0 \leqslant n \leqslant N-1$ 时间间隔之外为 0 的实的非奇序列. 假设 $x(0) \neq 0$, 并满足 $x(N-1)=-x(0)$, 那末仅用端点 $x(0)$ 以及幅度谱 $|X(\omega)|$ 不能唯一地恢复原始序列 $x(n)$, 它们至少还确定了另一个不同的序列 $y(n)$ ，并且满足关系式

$$
y(n)=-x(N-1-n) .
$$

关于该定理的证明,类似于定理 2,略.

\section{2. 下面讨论已知采样点是原始序列的非端点时的情形}

例如，仅仅已知采样值 $x(1)$ 和自相关 $R_{i}(0 \leqslant j \leqslant N-1)$, 要求唯一恢复原始序列 $x(n)$, 那末, 类似于定理 1 的证明,我们将不难得到定理 4. 但必须先把定义 1 改为定义 3 ,并仍用定 
义 2 .

定义 3. 令向量值函数 $F$ 为定义在区域 $\mathscr{D} \subset R^{N-1}$ 上而取值于 $R^{N}$ 的非线性实值函数. 简 记为

$F: \mathscr{D} \subset R^{N-1} \rightarrow R^{N}$, 它的具体形式为:

$$
F(X)=\left[f_{1}(X), f_{2}(X), \cdots, f_{N}(X)\right]^{T},
$$

其中 $X$ 为未知采样值 $x_{0}, x_{2}, x_{3}, \cdots, x_{N-1}$ 构成的向量 $X=\left[x_{0}, x_{2}, x_{3}, \cdots, x_{N-1}\right]^{T} \in R^{N-1}$; $f_{i}(X)(i=1,2, \cdots, N)$ 为定义在区域 $\mathscr{D} \subset R^{N-1}$ 上而取值于 $R^{1}$ 的非线性实值函数. 简记为 $f_{i}: \mathscr{D} \subset R^{N-1} \rightarrow R^{1}$, 它们的具体形式为

$$
\left\{\begin{array}{l}
f_{1}(X)=x_{0}^{2}+x_{1}^{2}+\cdots+x_{N-1}^{2}-R_{0}, \\
f_{2}(X)=x_{0} x_{1}+x_{1} x_{2}+\cdots+x_{N-2} x_{N-1}-R_{1}, \\
f_{3}(X)=x_{0} x_{2}+x_{1} x_{3}+\cdots+x_{N-3} x_{N-1}-R_{2}, \\
\cdots \cdots \cdots \\
f_{N-1}(X)=x_{0} x_{N-2}+x_{1} x_{N-1}-R_{N-2}, \\
f_{N}(X)=x_{0} x_{N-1}-R_{N-1} .
\end{array}\right.
$$

这里, $R_{j}(0 \leqslant j \leqslant N-1)$ 为序列 $x(n)$ 的自相关函数 (通常可由已知的幅度谱 $|X(\omega)|$ 的 逆 Fourier 变换求得); $x_{1}$ 为已知采样值; $x_{0}, x_{2}, x_{1}, \cdots, x_{N-1}$ 为序列 $x(n)$ 的末知采样值.

定理 4. 对于 $N>0$, 令 $x(n)$ 是一个在 $0 \leqslant n \leqslant N-1$ 时间间隔之外为 0 的实的混 合相位序列. 假设 $x(0) \neq 0$, 那末采样点 $x(1)$ 以及自相关 $R_{i}(0 \leqslant j \leqslant N-1)$ 唯一地确 定了整个序列 $x(n)$ ，当且仅当目标函数 $\varphi(X)$ 是凸集令 $\mathscr{D}_{j} \subset \mathscr{D}$ 上的严格凸函数.

当然, 类似于定义 3 和定理 4 , 我们也不难推广到仅仅已知采样值 $x$ (2) 或 $x(3), \cdots$, 或 $\cdot x(N-1)$ 的情形. 但不管是哪种情形, 唯一性条件的形式都与定理 1 相同,只不过未知函数 $f_{i}(X)$ 和末知向量 $X$ 各不相同而已.

\section{三、算法及实例}

在上一节中,我们已经规定了若干个条件, 在这组松弛的条件下, 仅用一个采样点以及自 相关函数就可以唯一地确定任意一个有限长度离散信号.

为了实现这种恢复，我们采用一种时域迭代算法。这种算法类似于 Himelblau 在 1972 年提出的可变多面体方法 ${ }^{[3]}$. 只要把已知采样点和自相关函数代入上节中的定义 1 (或 3) 和 定义 2 ,构造一个目标函数 $\varphi(X)$ (其中 $X$ 是 $\boldsymbol{n}$ 维向量), 然后用可变多面体方法寻找该目标函 数的极小值点. 当 $\varphi(X)$ 满足定理 1 或定理 4 规定的严凸条件时，这种迭代过程将会使多面 体的顶点逐步收敛到唯一的全局极小点, 即唯一地确定了整个离散信号. 我们在图 1 中给出 了这种时域迭代算法的程序框图。

用这种时域迭代算法来寻找目标函数的极小点，它的优点是稳定性好，准备时间短，因而 可以节省大量的人力. 同时, 它不要求目标函数具有连续性,因此适用的范围比较广. 这种方 法的缺点是程序收敛可能比较慢。

我们应用这种算法仅用端点 $x(0)$ 和自相关函数 $R_{1}(n)$ (图 2(b)) 成功地恢复了图 2(a)所 示的原始序列, 其结果见图 2(c). 由于不难证明这个原始序列满足定理 1 的严凸条件,因此 当我们改变一组初值后(如表 1), 仍然成功地实现了恢复. 所得到的估计序列(图 2(d)) 与原 


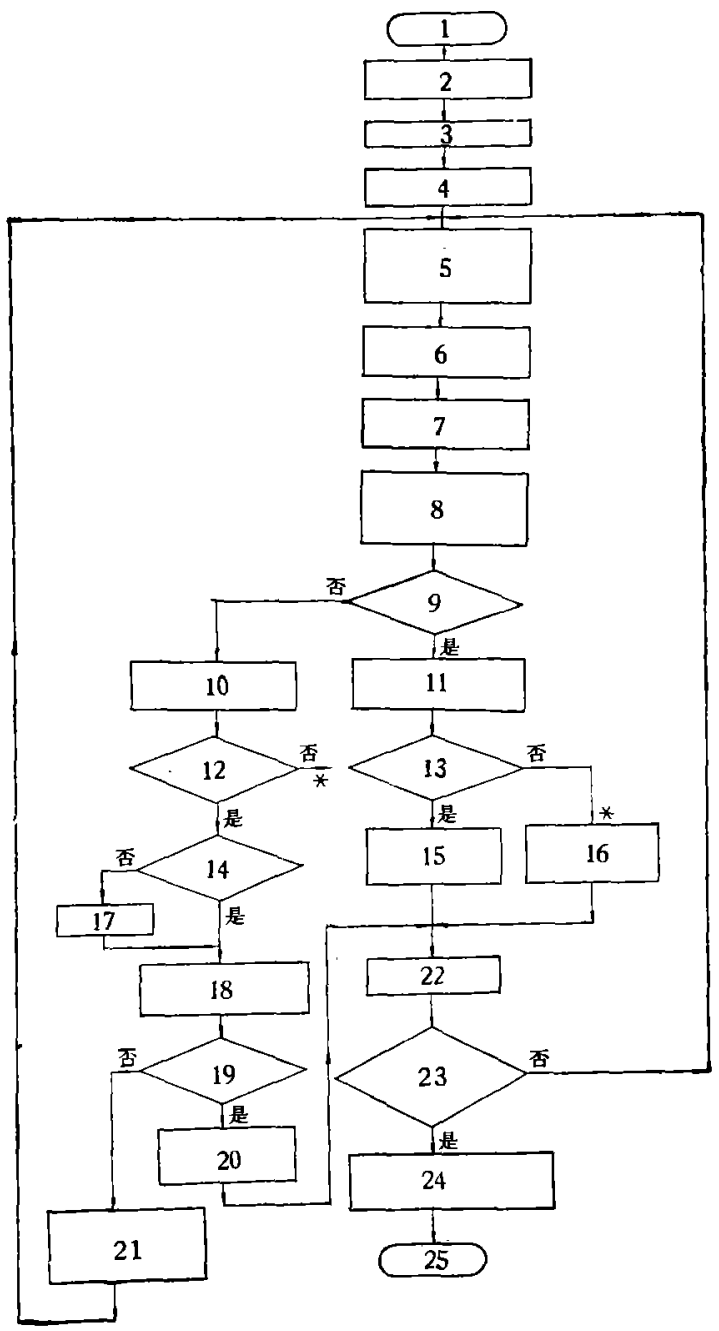

图 l 仅用一个采样点以及自相关饭复离散信号的程序框图

(1- - 启动, 2-一代入已知采样点和目相关构造目标函数 $\Phi(x), 3$ - 确定步长, 构造初始单纯形, 4- - 计算每个顶点 $x_{i}$ 的 $\Phi\left(x_{i}\right)$ 的值; $i=1,2, \ldots, n+1,5$ - 选取 $\Phi\left(x_{i}\right)$ 为最大的顶点 $x_{h}^{(k)}$, $\Phi\left(x_{h}^{(k)}\right)=\max \Phi\left(x_{i}^{(k)}\right), i=1, \ldots, n+1 ; k=0,1, \ldots, 6$ - - 选取 $\Phi\left(x_{i}\right)$ 为最小的顶点 $x_{i}^{(k)}$ $\Phi\left(x_{l}^{(k)}\right)=\min \Phi\left(x_{i}^{(k)}\right), 7$ - 计算除 $x_{h}^{(k)}$ 顶点外的顶点的重心 $x_{k+2}^{(k)}, 8$ - 映射: 将 $x_{h}^{(k)}$ 通过重 心 $x_{n+2}^{(k)}$ 映射到 $x_{n+3}^{(k)} ; x_{n+3}^{(k)}=x_{n+2}^{(k)}+\alpha\left(x_{n+2}^{(k)}-x_{k}^{(k)}\right)$, 其中 $\alpha>0,9-\Phi\left(x_{n+3}^{(k)}\right)<\Phi\left(x_{l}^{(k)}\right)$,

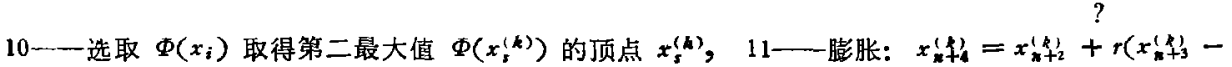
$\left.x_{n+2}^{(k)}\right)$, 其中 $\left.r>0,12-\Phi\left(x_{k+3}^{(k)}\right)\right\rangle \Phi\left(x_{s}^{(k)}\right), 13-\Phi\left(x_{n+4}^{(k)}\right)\left\langle\Phi\left(x_{k}^{(k)}\right), 14-\Phi\left(x_{n+3}^{(k)}\right)\right\rangle$ $?$ ?

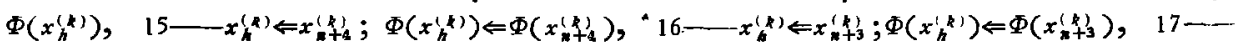
$x_{h}^{(k)} \Leftarrow x_{n}^{(k)}, 18$ - 收缩: $\quad x_{k+5}^{(k)}=x_{n+2}^{(k)}+\beta\left(x_{h}^{(k)}-x_{n+2}^{(k)}\right)$, 其中 $0<\beta<1,19--\Phi\left(x_{h}^{(k)}\right)>$ $\Phi\left(x_{n}^{(k)}\right), 20-x_{k}^{(k)} \Leftarrow x_{k+1}^{(k)} ; \Phi\left(x_{k}^{(k)}\right) \Leftarrow \Phi\left(x_{n}^{(k)}\right), 21$ 一将原单纯形缩小一半: $x_{i}^{(k+1)}=x_{i}^{(k)}+$ $0.5\left(x_{i}^{(k)}-x_{i}^{(k)}\right), t=1,2, \ldots, n+1,22-$ - 计算 $\Phi\left(x_{n+2}^{(k)}\right), 23-\left\{\frac{1}{n+1} \sum_{i=1}^{n+1}\left[\Phi\left(x_{i}^{(k)}\right)-\right.\right.$ $\left.\left.\Phi\left(x_{n}^{\prime} f_{2}^{\prime}\right)\right]^{2}\right\}^{\frac{1}{2}} \leqslant \varepsilon, 24-\Phi(x)$ 在极小值点的近似值 $X$ 处的值 $\Leftarrow \Phi\left(x_{2}^{\prime} f_{2}^{\prime}\right), 25$ - 结束)

始序列之间的误差仍然很小。这表明仅用端点 $x(0)$ 和自相关函数 $R_{\mathrm{t}}(n)$ 能唯一地确定原始 


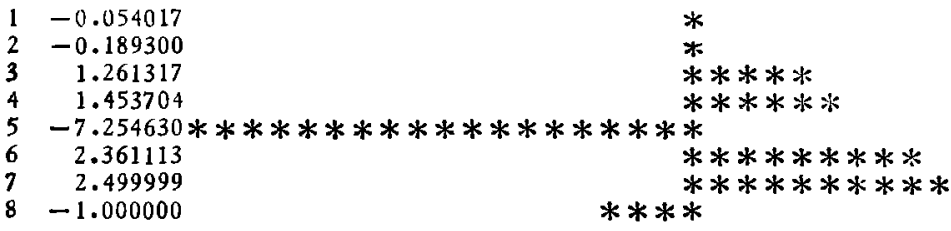

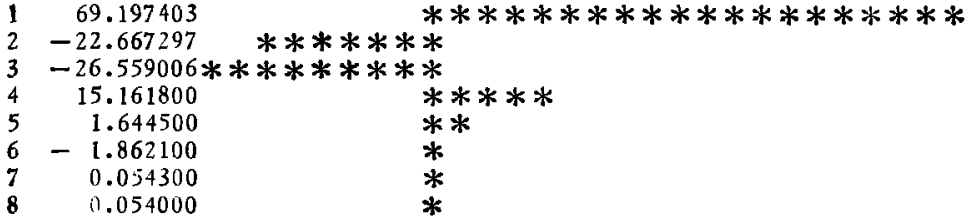

(b)

$\begin{array}{ccc}1 & -0.054012 & * \\ 2 & -0.188668 & * \\ 3 & 1.259675 & * * * * \\ 4 & 1.446428 & * * * * * \\ 5 & -7.253025 * * * * * * * * * * * * * * * * * * * * * \\ 6 & 2.370186 & * * * * * * * \\ 7 & 2.500257 & * * * * * * * * \\ 8 & -1.002247 & * * * *\end{array}$

(c)

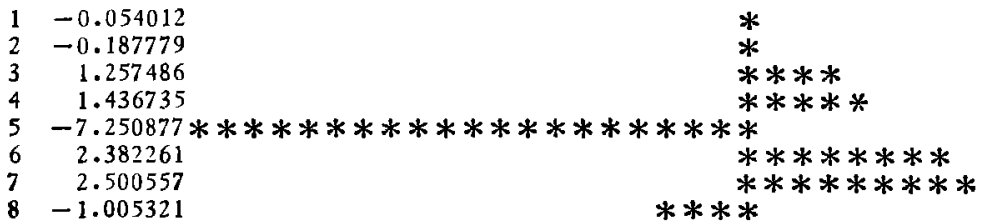

(d)

图 2 仅用端点以及自相关唯一恢复原始时间序列的一个例子

（(a) 原始序列 $(N=8)$ ，(b) 自相关序列 $R_{1}(j) ，(\mathrm{c})$ 仅用 $x(0)$ 和 $R_{1}(j)$ 恢复的

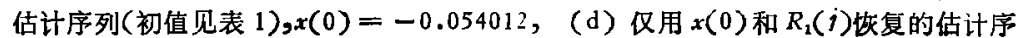
列（初值见表 1)）

$-0.324075 \quad * *$
$-2.486111 * * * * * * * *$
$-2.025717 \quad * * * * * * *$
$7.014408 * * * * * * * * * * * * * * * * * * * * * *$
$-2.308655 * * * * * * *$
$-1.736104 \quad * * * * * *$
$1.111110 \quad * * * *$
-0.166667

(a)

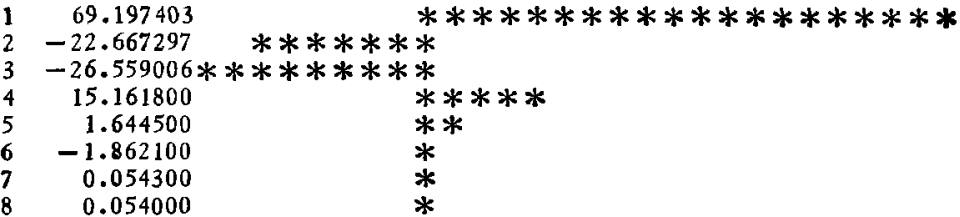


$-0.324075 * *$

$-2.486198 * * * * * * * *$

$-2.025877 * * * * * *$

7.014343
$-2.308617 * * * * * * * *$

$-2.308617 * * * * * * * *$
$-1.736118 * * * * * *$

$\begin{array}{rc}1.111073 & * * * * * \\ -0.166671 & *\end{array}$

(c)

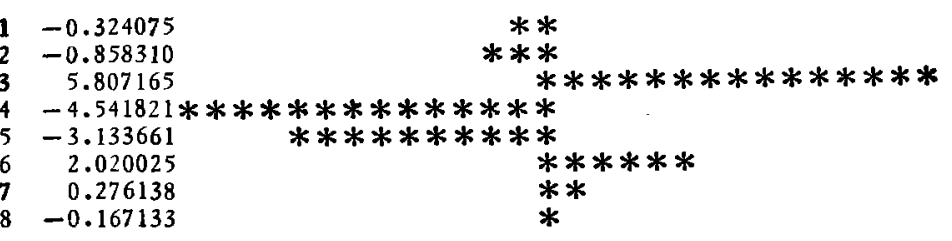

(d)

图 3 仅用端点以及自相关不能唯一恢复原始序列的一个例子

（(a) 原始序列 $(N=8)$, (b) 自相关序列 $R_{2}(1),(\mathrm{c})$ 仅用 $x(0)$ 和 $R_{2}(i)$ 恢复的估 计序列(初值见表 1), $x(0)=-0.324075$, (d) 仅用 $x(0)$ 和 $R_{2}(j)$ 败复的估计 序列（初值见表 1)）
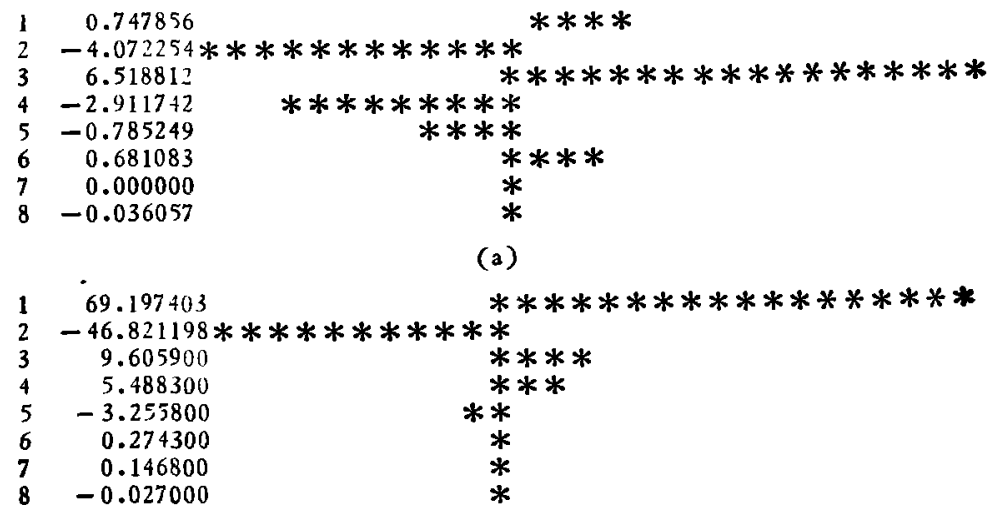

(b)

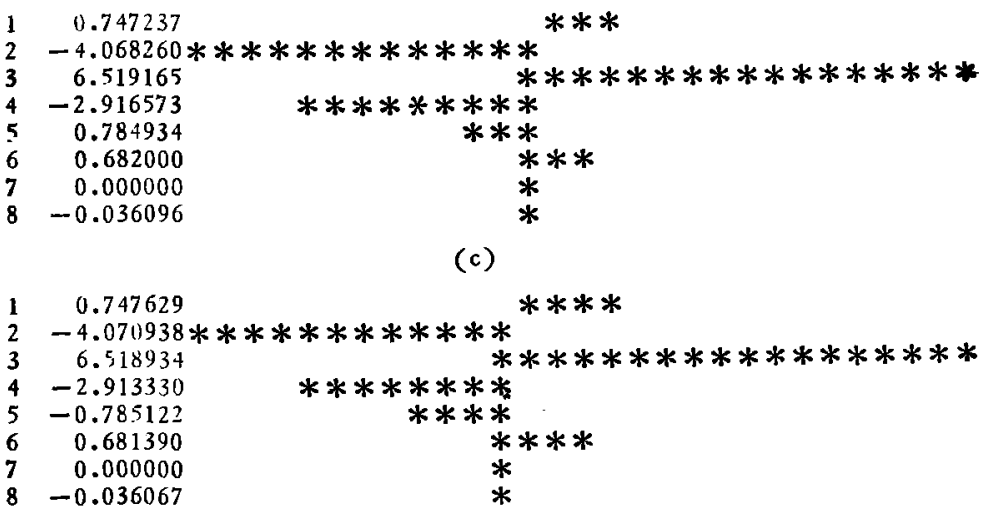

(d)

图 4 仅用一个采样点(非端点)以及自相关唯一恢复原始序列的一个例子 ((a) 原始序列 $(N=8)$ ，(b) 自相关序列 $R_{3}(i)$ ，(c) 仅用 $x(7)$ 和 $R_{3}(i)$ 奴复的 估计序列(初值见表 1 ), $x(7)=0.0$, (d) 仅用 $x(7)$ 和 $R_{3}(i)$ 败复的估计序列( 和 宣见表 1$), x(7)=0.0$ ) 


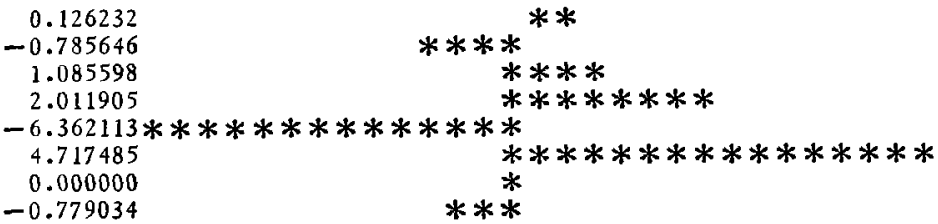

(a)

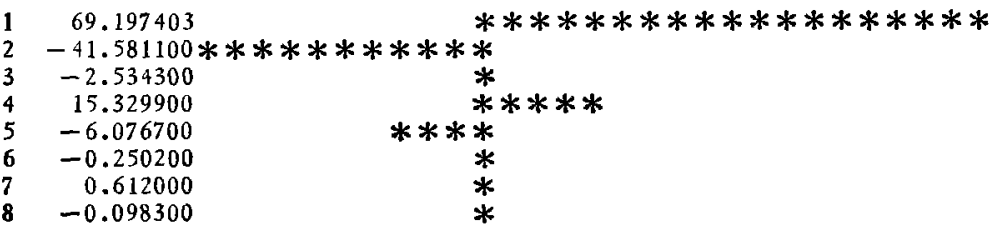

(b)

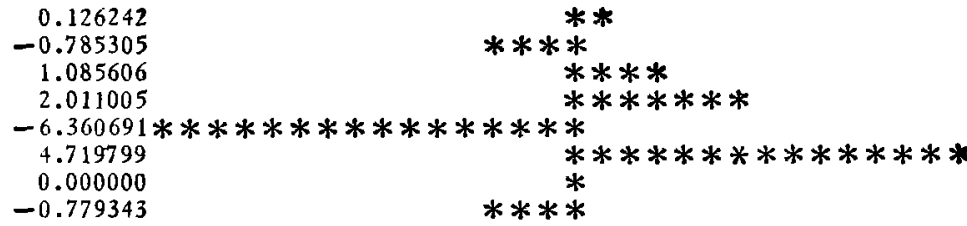

(c)

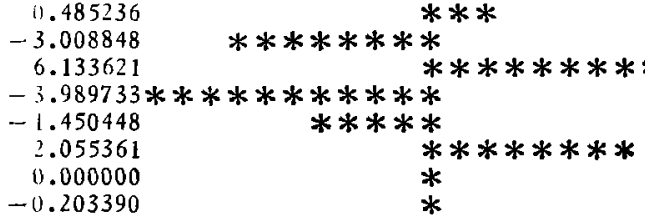

(d)

图 5 仅用一个采样点(非端点)以及自相关不能唯一恢复原始序列的一个例子

((a) 原始序列 $(N=8)$ ，(b) 自相关序列 $R_{4}(i)$ ，(c) 仅用 $x(7)$ 和 $R_{4}(i)$ 恢复的估计序列（初值见 (表 1)，x(7)=0.0，(d)仅用 $x(7)$ 和 $R_{4}(i)$ 炍复的估计序列（初值见表 1)， $\left.x(7)=0.0\right)$ ）

疗列(图(a)),而与初值选择无关.

\begin{tabular}{|c|c|c|c|c|c|c|c|c|c|}
\hline 数 & \multicolumn{8}{|c|}{ 迭 代初 值 } & \multirow{2}{*}{ 步长 } \\
\hline & $x(1)$ & $x(2)$ & $x(3)$ & $x(4)$ & $x(5)$ & $x(6)$ & $x(7)$ & $x(8)$ & \\
\hline $2(c)$ & -0.054 & 1.0 & 5.0 & -5.0 & -10.0 & 5.0 & 5.0 & -1.0 & 4.4 \\
\hline $2(d)$ & -0.054 & 0.0 & 1.0 & 1.0 & 0.0 & 1.0 & 1.0 & 0.0 & 4.4 \\
\hline $3(c)$ & -0.324 & -1.0 & 5.0 & -5.0 & -5.0 & 5.0 & 1.0 & -1.0 & $\begin{array}{l}5.0 \\
8.0\end{array}$ \\
\hline $3(d)$ & -0.324 & -5.0 & -5.0 & 10.0 & -5.0 & -1.0 & 1.0 & -1.0 & 4.4 \\
\hline $4(c)$ & 1.0 & -5.0 & 10.0 & -5.0 & -1.0 & 1.0 & 0.0 & -1.0 & 4.84 \\
\hline $4(d)$ & 5.0 & -10.0 & 5.0 & -1.0 & 0.0 & -1.0 & 0.0 & 1.0 & 8.0 \\
\hline $5(c)$ & 1.0 & -1.0 & 2.0 & 5.0 & -10.0 & 5.0 & 0.0 & -1.0 & $\begin{array}{l}4.98 \\
5.04\end{array}$ \\
\hline $5(d)$ & 1.0 & -5.0 & 10.0 & -5.0 & -1.0 & 1.0 & 0.0 & -1.0 & 5.0 \\
\hline
\end{tabular}


与此相反,当我们取两组不同的初值时（如表 1 ），仅用端点 $x(0)$ 和自相关函数 $R_{2}(n)$ (图 3(b) ) 却不能唯一恢复图 3(a) 所示的原始序列. 其结果如图 3(c) 和 (d) 所示. 这是因为, 不难证明图 3(a) 所示的原始序列仅仅是凸函数,而不满足定理 1 规定的严凸条件. 所以，由 该序列的端点 $x(0)$ 和它的自相关函数 $R_{2}(n)$ 所构造的目标函数 $p_{2}(X)$ 的任一局部极小点必 为全局极小点，但不是唯一的 ${ }^{61}$. 正如图 3(d) 所示的那样，尽管该估计序列与原始序列之间 有着很大的差别,然而它也使目标函数 $\varphi_{2}(X)$ 的极小值非常接近于 0 (见表 1)。这说明不但 图3(c) 所示的估计序列是 $\varphi_{2}(X)$ 的极小点, 而且图 3(d) 所示的估计序列也是该 $\varphi_{2}(X)$ 的极 小点. 也就是说, $\varphi_{2}(X)$ 至少存在着两个或两个以上的全局极小点.

类似地,我们还应用这种时域迭代算法，仅用一个采样点(非端点)和自相关函数成功地恢 复了原始序列. 如图 4(a) 中给出了一个原始序列 $(N=8)$. 假设已知采样点为 $x(7)$, 该未知 序列的自相关 $R_{3}(n)$ 为图 4(b) 所示. 可以证明, 由 $x(7)$ 和 $R_{2}(n)$ 所构造的目标函数 $\varphi_{3}(X)$ 满 足类似于定理 4 的严凸条件，因此当我们用两组不同的初值时(如表 1)，选代所得到的两个估 计序列(如图 4(c) 和(d)) 都非常接近于原始序列。这表明仅用一个采样点 $x(7)$ 和自相关函 数 $R_{3}(n)$ 能唯一地确定原始序列（图 4(a)), 而与初值选择无关. 另外, 令人感兴趣的是由于 已知采样点非常靠近于原始序列的尾部，因此若用时域一频域迭代算法 ${ }^{\text {山l }}$ ，对于这个实例却 显得无能为力,无法收玫于原始序列, 而用本节介绍的时域迭代法却能十分成功地恢复原始序 列。这说明这种算法的稳定性要优于时域一一频域迭代法.

我们在图 5(a)中还给出了另一个原始序列 $(N=8)$. 假设已知采样点与例 3 相同, 仍然为 $x(7)$, 但自相关 $R_{4}(n)$ 却不同于例 3, 如图 5(b) 所示,因此可得到一个不同于 $\varphi_{3}(X)$ 的目标函 数 $\varphi_{4}(X)$. 可以证明, 这个 $\varphi_{4}(X)$ 并不满足类似于定理 4 的严凸条件. 当我们用两组不同的 初值时 (如表 1), 选代所得到的两个估计序列虽然使 $\varphi_{4}(X)$ 的极小值都非常接近于零,然而其 中一个估计序列(图 5(c)) 与原始序列之间十分接近,而另一个估计序列(图 5(d)) 与原始序列 之间却有着很大的差异（见表 1)。这表明由非端点 $x(7)$ 和自相关函数 $R_{4}(n)$ 所构造的目标函 数 $\varphi_{4}(X)$ 至少存在着两个或两个以上的全局极小点. 因此, 仅用非端点 $x(7)$ 和自相关函数 $R_{4}(n)$ 是不能唯一确定原始序列(图 5(d)) 的.

1

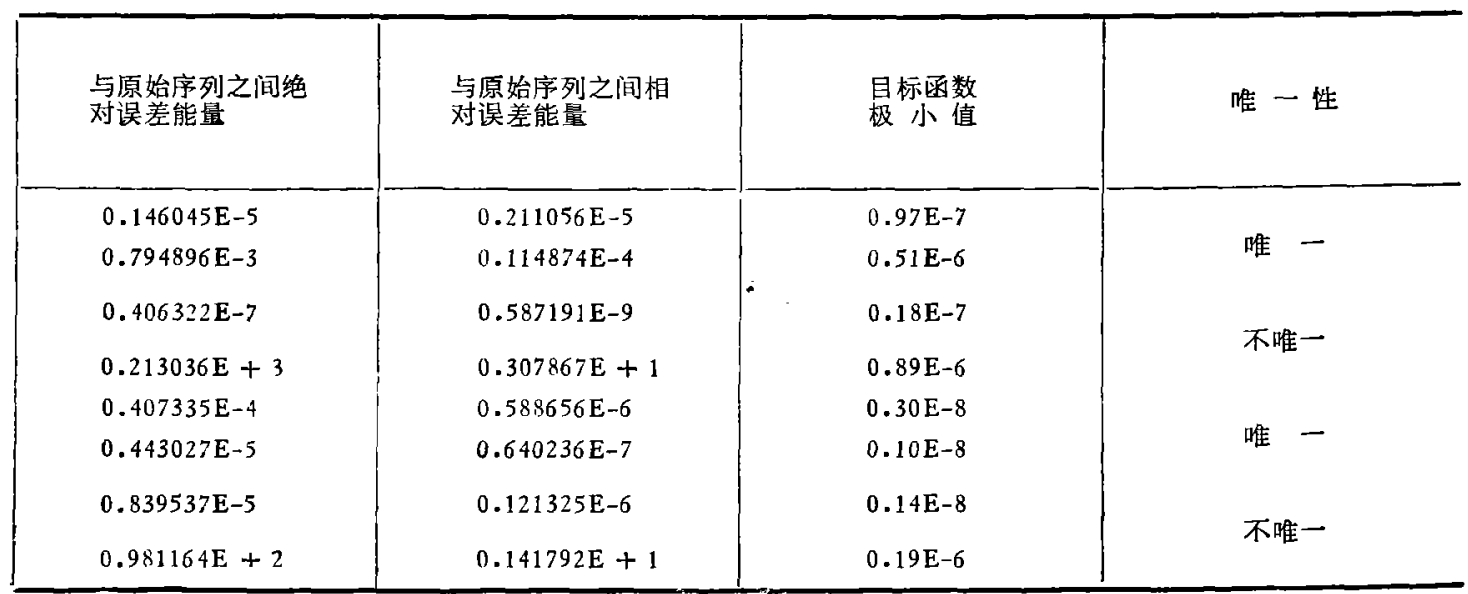




\section{四、结论}

通过本文的理论分析以及大量的实例验证，我们不难得出以下几点结论:

第一, 只要满足一组松弛的条件,那末恢复非最小相位信号所需的已知段的长度 $m$ 就可以 缩小到 $m=1$, 即仅仅由一个已知采样点以及自相关函数就能唯一地确定任意一个离散信 号, 而且这个已知采样点可以是未知序列中的任何一个采样点。这不但大大扩展了我们在文 献[1]中提出的定理 3一定理 5 的适用范围, 而且更重要的是从理论上较为圆满地解决了本文 引论中,所提出的关于利用部分信息恢复有限长度离散信号的第一个基本问题。也就是说，如 果仅仅依赖于幅谱这一部分信息来恢复非最小相位信号, 必然不满足唯一性. 为此, 只需增加 一点信息, 即已知时域中的任何一个采样点, 当这个已知采样点和自相关函数所构成的目标 函数（由定义 1 或 3 和定义 2) 为严凸函数时，那末仅由幅谱就能唯一地恢复非最小相位离 散信号.

第二, 为了实现仅由一个时域采样点以及自相关恢复离散信号, 可以采用类似于可变多 面体搜索方法的时域迭代法. 实例表明, 这种算法是十分有效的. 与时域-频域迭代法相比 较, 虽然两种算法都是引入了全部的非线性方程作为约束条件,然而, 由于时域迭代法省去了 时频域变换这一步聚，因此计算时间大大缩短，稳定性也显著提高. 从而进一步解决了本文引 论中所提出的关于利用信息恢复信号的第二个基本问题。当然在应用这种算法时, 必须选择 合适的迭代初值和初始多面体的步长,否则就不易收玫于我们要寻找的那些未知的样本点. 另 外,在某些情况下，由于目标函数的严凸性条件可能难于验证，因此利用这种时域迭代法求得 的极小点,只能认为是局部极小点. 在实际计算中, 可以选择不同的初始近似进行搜索, 将所 得的局部极小点进行比较, 取其中使目标函数值为最小的那个局部极小点作为所求的结果。这 种作法在解决实际问题过程中常常是有效的.

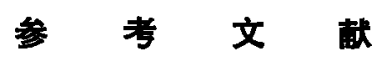

[1] 吴忠泽、李衍达、常迥,中国科学 A 辑，1986,10：1109.

[2] Enders, A. R. and Sven Treitel, Geophysical Signal Analysis, Prentice-Hall, Inc., Englewood Cliffs, N. J.r $1980,07632$.

[ 3 ] Nawab, S. H., Quatieri, T. F. \& Lim, J. S., IEEE, ASSP-31 (1983), 4: 997-1000.

[4] 数学手册, 人民教育出版社, 1979,92.

[ 5 ] Himeblau, D. M., Applied Nonlinear Programming, Mcgrawhill Book Company, N. Y., 1972.

[6]王德人,非线性方程解法与最优化方法,人民教育出版社，1979. 